\title{
A Krull-Schmdit type theorem for coherent sheaves
}

\author{
A. S. Argáez
}

\begin{abstract}
Let $X$ be projective variety over an algebraically closed field $k$ and $G$ be a finite group with g.c.d. $(\operatorname{char}(k),|G|)=1$. We prove that any representations of $G$ on a coherent sheaf, $\rho: G \longrightarrow \operatorname{End}(\varepsilon)$, has a natural decomposition $\mathcal{E} \simeq \bigoplus V \otimes_{k} \mathcal{F}_{V}$, where $G$ acts trivially on $\mathcal{F}_{V}$ and the sum run over all irreducible representations of $G$ over $k$.
\end{abstract}

\section{Introduction}

Let $X$ be a complete variety over an algebraically closed field $k$ and let $G$ be a finite group with $\operatorname{char}(k)$ and $|G|$ coprimes, thus the $k$-algebra $k[G]$ is semisimple (not necessary commutative) of finite dimension $|G|$ over $k$. Let $\mathcal{O}_{X}[G]:=\mathcal{O}_{X} \otimes_{k} k[G]$. Thus, we can define the category A where the objects are $\mathcal{O}_{X}[G]$-modules, which are defined as pairs consisting of an $\mathcal{O}_{X}$-module $\mathcal{E}$ together with a $k$-morphism of rings $\rho: k[G] \rightarrow \operatorname{End}(\mathcal{E})$ and the morphisms are defined in the natural way. Clearly this an Abelian category. We say that an $\mathcal{O}_{X}[G]$-module $\mathcal{E}$ is indecomposable if every direct decomposition of $\mathcal{E}$ into $\mathcal{O}_{X}[G]$-modules is trivial. From the Krull-Schmidt Theorem proved by Atiyah in [1], on a complete variety $X$ every non-zero $\mathcal{O}_{X}[G]$-module $\mathcal{E}$ has a direct sum decomposition into indecomposable $\mathcal{O}_{X}[G]$-modules and this decomposition is unique up to permutations. The objective of this paper is to prove the next structure theorem for $\mathcal{O}_{X}[G]$-modules.

\footnotetext{
Key Words: Group representations on sheaves, Krull-Schmdit theorem, projective varieties.

2010 Mathematics Subject Classification: Primary 20F29; Secondary: 14A25

Received: April, 2012.

Accepted: February, 2013
} 
Theorem 1. Let $X$ be a complete variety over an algebraically closed field $k$. Let $\mathcal{E}$ be a coherent $\mathcal{O}_{X}[G]$-module and suppose there is a surjective $G$ morphism

$$
\mathcal{F} \longrightarrow \mathcal{E} \longrightarrow 0
$$

with $\mathcal{F}$ torsion free. Let $V_{0}, \ldots V_{r}$ be the irreducible representations of $G$ over $k$. Then, there is a natural $\mathcal{O}_{X}[G]$-isomorphism

$$
\mathcal{E} \simeq\left(V_{0} \otimes_{k} \mathcal{E}_{V_{0}}\right) \oplus \ldots \oplus\left(V_{r} \otimes_{k} \mathcal{E}_{V_{r}}\right)
$$

where the action of $G$ on $\mathcal{E}_{V_{i}}$ is trivial for all $i$. In particular, if $X$ is a projective variety, this is true for any coherent $\mathcal{O}_{X}[G]$-module.

\section{Proof of Theorem 1}

Lemma 1. Let $k$ be an algebraically closed field and $G$ be a finite group. Then, for any field extension $K$ of $k$, any $K$-representation of $G$ is of the form $V \otimes_{k} K$ with $V$ a k-representation of $G$, unique up to $G$-isomorphisms.

Proof. It is sufficient to prove the lemma for irreducible representations. By the corollary 3.61 in [2] page 68, any representation of the form $V \otimes_{k} K$ is irreducible over $K$ if $V$ is irreducible over $k$, and by theorem 30.15 in [3] page 214 all those are different irreducible representations, then those are all the irreducible representations.

Definition 1. Let $X$ be an integral scheme over an algebraically closed field $k, K$ be the function field of $X$ and $\epsilon$ be the generic point. Let $\mathcal{E}$ be a torsion free coherent $\mathcal{O}_{X}[G]$-module, and $\mathcal{E}_{\epsilon} \simeq V \otimes_{k} K$ be the representation of $G$ at the generic point, we define the type of the representation of $G$ on $\mathcal{E}$ as the isomorphisms class of the representation $V$. Also, if $\mathcal{G}$ is any $\mathcal{O}_{X}[G]$-module, we define the isotypical decomposition of $\mathcal{G}$ by the decomposition

$$
\mathcal{G}=e_{0} \mathcal{G} \oplus \ldots \oplus e_{r} \mathcal{G}
$$

where $\left\{e_{i}\right\}$ are the respective idempotents of $k[G]$. Notice that $e_{V}$ define an exact functor from $\mathcal{A}$ to $\mathcal{A}$.

Now, the type of the representation and the isotypical decomposition are related by 
Lemma 2. Let $X$ be a variety over an algebraically closed field $k, K$ be its function field and $\epsilon$ be the generic point. Let $\mathcal{E}$ be a torsion free coherent $\mathcal{O}_{X}[G]$-module of type $V$. Then

$$
e_{i}\left(\mathcal{E}_{\epsilon}\right)=e_{i}\left(V \otimes_{k} K\right)=\left(e_{i} \mathcal{E}\right)_{\epsilon}
$$

where $e_{i}$ is the idempotent element on $k[G]$ corresponding to the representation $V_{i}$. Furthermore, the isotytpical decomposition of the $K[G]$-module $\mathcal{E}_{\epsilon}$ is given by

$$
\mathcal{E}_{\epsilon}=V \otimes_{k} K=\bigoplus_{i=0}^{r}\left(e_{i} \mathcal{E}\right)_{\epsilon}
$$

Proof. The first part of the theorem is an immediately consequence of the properties of the stalk at a point. The second part follows from lemma 1.

Lemma 3. (Characterization of free torsion $\mathcal{O}_{X}[G]$-modules) Let $X$ be a complete variety over an algebraically closed field $k$, let $\mathcal{E}$ be an indecomposable torsion free coherent $\mathcal{O}_{X}[G]$-module of $W$ type. Then $\mathcal{E} \simeq V \otimes_{k} \mathcal{F}$ with $\mathcal{F}$ an indecomposable $\mathcal{O}_{X}$-module and $W \simeq V^{\oplus r a n k \mathcal{F}}$, with $V$ an irreducible representation.

In particular, if $\mathcal{G}$ is a torsion free coherent $\mathcal{O}_{X}[G]$-module of type $V_{0}^{n_{0}} \oplus$ $\ldots \oplus V_{r}^{n_{r}}$. Then the isotypical decomposition of $\mathcal{G}$ is given by

$$
\mathcal{G} \simeq\left(V_{0} \otimes_{k} \mathcal{G}_{V_{0}}\right) \oplus \ldots \oplus\left(V_{r} \otimes_{k} \mathcal{G}_{V_{r}}\right)
$$

where $\mathcal{G}_{V_{i}}$ is an $\mathcal{O}_{X}$-module of rank $n_{i}$.

Proof. As $X$ is a complete variety, we can apply the Krull-Schmidt theorem for coherent sheaves proved in [1]. Thus, let $\mathcal{E}=\mathcal{F}_{1}^{\oplus n_{1}} \oplus \ldots \oplus \mathcal{F}_{r}^{\oplus n_{r}}$ be the unique decomposition of $\mathcal{E}$ into indecomposable $\mathcal{O}_{X}$-modules with $\mathcal{F}_{i} \neq \mathcal{F}_{j}$, if $i \neq j$. Hence, if $g \in G, g \mathcal{E}=g \mathcal{F}_{1}^{\oplus n_{1}} \oplus \ldots \oplus g \mathcal{F}_{r}^{\oplus n_{r}}$ imply $g \mathcal{F}_{i}=\mathcal{F}_{j}$ for some $j$, then $\mathcal{F}_{i} \simeq \mathcal{F}_{j}$ and $i=j$. Now, any $\mathcal{F}_{i}^{\oplus n_{i}}$ must be $G$-invariant but $\mathcal{E}$ is an indecomposable $\mathcal{O}_{X}[G]$-module so $r=1$ and $\mathcal{E}=\mathcal{F}^{\oplus n}$ with $\mathcal{F}$ an indecomposable $\mathcal{O}_{X}$-module.

Let $W$ the type of the representation, therefore, $W \simeq V^{\oplus s}$ with $V$ irreducible. Thus, the next step is to show that $s=\operatorname{rank} \mathcal{F}$ and $\operatorname{dim} V=n$. For this, we consider the part of type $V_{0}$ of $V^{\vee} \otimes_{k} \mathcal{E}$, where $V^{\vee}$ is the dual representation of $V$, this is a direct summand of $V^{\vee} \otimes_{k} \mathcal{E} \simeq \mathcal{F}^{\oplus n \cdot \operatorname{dim} V}$, so this component must be $\mathcal{F}^{\oplus i}$ for some $i$. Now, taking the composition of the canonical inclusion of $\mathcal{O}_{X}[G]$-module

$$
V \otimes_{k} \mathcal{F}^{\oplus i} \simeq\left(V \otimes_{k} \mathcal{F}\right)^{\oplus i} \longrightarrow\left(V \otimes_{k} V^{\vee}\right) \otimes_{k} \mathcal{E}
$$


with the canonical map

$$
\left(V \otimes_{k} V^{\vee}\right) \otimes_{k} \mathcal{E} \longrightarrow \mathcal{E}
$$

we have an $\mathcal{O}_{X}[G]$-morphism

$$
\alpha:\left(V \otimes_{k} \mathcal{F}\right)^{\oplus i} \longrightarrow \mathcal{E} \simeq \mathcal{F}^{\oplus n}
$$

that is a $K[G]$-isomorphism at the generic point, so $n=i \cdot \operatorname{dim}(V)$. Now, $\mathcal{F}$ is torsion free, so $\alpha$ must be injective and we can apply the last corollary in [1], this corollary claim that, over a complete variety, an injective endomorphism is an isomorphism, but we are supposing that $\mathcal{E}$ is an indecomposable $\mathcal{O}_{X}[G]$ module, then $i=1$ and $\mathcal{E} \simeq V \otimes_{k} \mathcal{F}$ and the theorem follows.

Now we have

Proof of Theorem 1 From the hypotheses we have and exact sequence of $\mathcal{O}_{X}[G]$-modules

$$
0 \longrightarrow \mathcal{K} \longrightarrow \mathcal{F} \longrightarrow \mathcal{E} \longrightarrow 0
$$

with $\mathcal{F}$ a coherent torsion free sheaf, thus we have that $\mathcal{K}$ is torsion free.

On the other hand, for each $T \in \mathcal{J}$ we have an exact sequence

$$
0 \longrightarrow e_{T}(\mathcal{K}) \longrightarrow e_{T}(\mathcal{F}) \longrightarrow e_{T}(\mathcal{E}) \longrightarrow 0
$$

and from Lemma 2 above, there are unique sheaves $\mathcal{K}_{T}$ and $\mathcal{F}_{T}$ such that $e_{T}(\mathcal{K}) \simeq T \otimes \mathcal{K}_{T}$ and $e_{T}(\mathcal{F}) \simeq T \otimes \mathcal{F}_{T}$, thus we have the exact sequence

$$
0 \longrightarrow T \otimes_{k} \mathcal{K}_{T} \longrightarrow T \otimes_{k} \mathcal{F}_{T} \longrightarrow e_{T}(\mathcal{E}) \longrightarrow 0
$$

applying the exact functor $e_{0}\left(T^{\vee} \otimes_{k}\right)$ we obtain

$$
0 \longrightarrow \mathcal{K}_{T} \longrightarrow \mathcal{F}_{T} \longrightarrow e_{0}\left(T^{\vee} \otimes_{k} e_{T}(\mathcal{E})\right) \longrightarrow 0
$$

and applying $T \otimes_{k-}$

$$
0 \longrightarrow T \otimes_{k} \mathcal{K}_{T} \longrightarrow T \otimes_{k} \mathcal{F}_{T} \longrightarrow T \otimes_{k} e_{0}\left(T^{\vee} \otimes_{k} e_{T}(\mathcal{E})\right) \longrightarrow 0
$$

but the first morphism in sequences 1 and 2 are the same, then both have the same cokernel, then $e_{T}(\mathcal{E}) \simeq T \otimes_{k} e_{0}\left(T^{\vee} \otimes_{k} e_{T}(\mathcal{E})\right)$ and the first part of the Theorem is proved.

On the other hand, if $X$ is projective, for any coherent sheaf $\mathcal{E}$ there is an exact sequence

$$
\widehat{\mathcal{F}} \stackrel{\widehat{\phi}}{\longrightarrow} \mathcal{E} \longrightarrow 0
$$


of $\mathcal{O}_{X}$-modules with $\widehat{\mathcal{F}}$ locally free (see [5] II 5.18). Therefore, when $\mathcal{E}$ is an $\mathcal{O}_{X}[G]$-module, we can construct an exact sequence of $\mathcal{O}_{X}[G]$-modules

$$
\mathcal{F} \stackrel{\phi}{\longrightarrow} \mathcal{E} \longrightarrow 0
$$

with $\mathcal{F}=k[G] \otimes \widehat{\mathcal{F}}, \phi\left(\sum a \cdot g\right) \otimes f \mapsto \sum a \cdot g \widehat{\phi}(f)$. Then the Theorem follows.

\section{References}

[1] M. F. Atiyah On the Krull-Schmidt theorem with applications to sheaves Bulletin de la S. M. F.,tome 84 (1956), p.307-317.

[2] C. W. Curtis \& I. Reiner, Methods of representation theory I, Wiley, New York, 1981.

[3] C. W. Curtis \& I. Reiner, Representation theory of finite groups and associative algebras Wiley, 1962.

[4] W. Fulton \& J. Harris, Representation theory: A first course, SpringerVerlag. 1991.

[5] Harstshorne R. Algebraic Geometry, Springer-Verlag, New York.

A. S. Argáez

Department of Mathematics,

Universida Autónoma "Benito Juárez" de Oaxaca,

Av Universidad s/n Oaxaca de Juárez, México.

Email: as_argaez@yahoo.com.mx 
